\title{
Charge profile in vortices
}

\author{
Jan Koláček, Pavel Lipavský \\ Institute of Physics, ASCR, Cukrovarnická 10, 16253 Prague 6, Czech Republic \\ Ernst Helmut Brandt \\ Max-Planck-Institut für Metallforschung, D-70506 Stuttgart, Germany
}

\begin{abstract}
The electric charge density in the vortex lattice of superconductors is studied within the GinzburgLandau theory. We show that the electrostatic potential $\varphi$ is proportional to the GL function, $\varphi \propto|\psi|^{2}-\left|\psi_{\infty}\right|^{2}$. Numerical results for the triangular vortex lattice are presented.
\end{abstract}

Abrikosov vortices in type-II superconductor consist of magnetic flux encircled by a rotating condensate of Cooper pairs. The inertial and Lorentz forces both act in the centrifugal direction and thus cause a small depletion of the charges in the vortex core. The electrostatic potential generated by this depleted charge supplies a centripetal force which balances the centrifugal force [1]:2].

While the depleted charge occupies a region comparable with the vortex core, i.e. a region extending over the Ginzburg-Landau (GL) coherence length $\xi$, the screening charge extends over a region extending over half the London penetration depth $\lambda$. This charge distribution results in an electrostatic field which may be observed either by nuclear quadrupole resonance 3 or by surface scanning as proposed recently by Blatter et al. 任 or by the Torricelli-Bernoulli effect [5]. Besides, the charge of the vortex core is expected to contribute to the forces acting on the vortex. For example, Feigel'man et al. [6] speculate that this charge can explain the still puzzling sign reversal of the Hall voltage.

The charge in the asymptotic region away from the vortex core has been studied by LeBlanc [2]. In this region one can benefit from the simplicity of the London theory. Within the London theory, the local balance of forces acting on the Cooper pair may be written in form of the Newton equation,

$$
m^{*} \dot{\mathbf{v}}=-e^{*} \nabla \varphi+e^{*} \mathbf{v} \times \mathbf{B} .
$$

Here the mass $m^{*}=2 m$ and charge $e^{*}=2 e$ are twice the values of a single electron. Unlike in common applications of the Newton equation (11) here the velocity is given by the London relation, $m \mathbf{v}=-e \mathbf{A}$, and Eq. (1) thus specifies the electric field $-\nabla \varphi$ which maintains this motion. Using the identity $\dot{\mathbf{v}}=(\mathbf{v} \nabla) \mathbf{v}=\frac{1}{2} \nabla\left(\mathbf{v}^{2}\right)-\mathbf{v} \times \nabla \times \mathbf{v}$ and the London approximation $e \mathbf{B}=-m \nabla \times \mathbf{v}$, one can show that Eq. (1) is solved by the Bernoulli potential,

$$
\varphi=-\frac{m v^{2}}{2 e}
$$

The quadratic dependence on the velocity shows that the electric potential $\varphi$ decreases over a characteristic length $\lambda / 2$. The charge density $\rho=-\varepsilon \nabla^{2} \varphi$ behaves similarly.

Obviously, the Bernoulli potential (2) is not suited for the core region, where the local London approximation fails. Moreover, close to the core the velocity diverges, $v \sim \frac{e \Phi_{0}}{2 \pi m} \frac{1}{r}$, where $\Phi_{0}$ is the quantum of flux and $r$ is the distance from the vortex axis. According to Eq. (2) and $\rho=-\varepsilon \nabla^{2} \varphi$, the total screening charge follows this divergence, $\int_{r}^{\infty} \mathrm{d} r \rho \propto r^{-2}$. The charge of the core estimated from the London expression (2) thus crucially depends on the estimate of the core diameter and the profile of the charge is incorrect in the core region.

In the core region, a non-local approach like the Ginzburg-Landau theory is required [7]. Like the London approach, the Ginzburg-Landau theory does not include the electrostatic potential explicitly. Here we show how this potential can be determined from its effect on the motion of Cooper pairs. The Bernoulli potential then has to be replaced by the potential

$$
\varphi=\frac{\beta}{2 e}\left(|\psi|^{2}-\left|\psi_{\infty}\right|^{2}\right),
$$

with $\beta$ and $\psi_{\infty}$ having the standard meaning in the Ginzburg-Landau theory [8].

The proof of (3) parallels the proof of the Bernoulli potential. The Cooper pairs move according to the Schrödinger equation,

$$
\frac{1}{2 m^{*}}\left(i \hbar \nabla+e^{*} \mathbf{A}\right)^{2} \psi+e^{*} \varphi \psi=0,
$$

which replaces the force balance equation (11).

The electrostatic potential $\varphi$ also causes a mismatch between the local Fermi level and the global chemical potential that results in a local suppression of Cooper pairs. Briefly, the charge densities of superconducting and normal electrons have a ratio different from the unperturbed state, $\rho_{\mathrm{s}}: \rho_{\mathrm{n}} \neq \rho_{\mathrm{s}}^{\infty}: \rho_{\mathrm{n}}^{\infty}$, where the superscript $\infty$ denotes values far from the perturbation. Due to this mismatch, the share of normal electrons is locally increased. In the linear approximation one has

$$
\rho_{\mathrm{n}}=\rho_{\mathrm{n}}^{\infty}-\frac{e^{2}}{D} \varphi .
$$

Taking the gradient, one can read (5) as balance between the electric force and a kind of osmotic pressure of normal electrons, $e \mathbf{E}=D \nabla\left(\rho_{\mathrm{n}} / e\right)$. In this sense, equation (5) describes the response of normal electrons to the electric 
field, importance of which has already been noticed by London [1]. The "diffusion coefficient" $D$ is identified below.

Finally, the electrostatic potential obeys the Poisson equation,

$$
-\varepsilon \nabla^{2} \varphi=\rho=\rho_{\mathrm{s}}+\rho_{\mathrm{n}}+\rho_{\text {lattice }} .
$$

With the wave function normalized to the charge density of the condensate, $\rho_{\mathrm{s}}=e^{*}|\psi|^{2}$, we can use the condition of neutrality, $e^{*}\left|\psi_{\infty}\right|^{2}+\rho_{\mathrm{n}}^{\infty}+\rho_{\text {lattice }}=0$, to eliminate the normal density from the Poisson equation,

$$
-\varepsilon \nabla^{2} \varphi=e^{*}\left(|\psi|^{2}-\left|\psi_{\infty}\right|^{2}\right)-\frac{e^{2}}{D} \varphi .
$$

The set of equations (4) and (7) is closed by the Maxwell equation, $\nabla \times \nabla \times \mathbf{A}=\mu \mathbf{j}$, with the superconducting current $\mathbf{j}=-\frac{e^{*}}{m^{*}} \operatorname{Re}\left[\bar{\psi}\left(i \hbar \nabla+e^{*} \mathbf{A}\right) \psi\right]$.

Let us suppose that the system is nearly neutral so that the left hand side of (7) may be disregarded. In this case, the potential can be determined from the suppression of the superconducting density,

$$
\varphi \approx \frac{2 D}{e}\left(|\psi|^{2}-\left|\psi_{\infty}\right|^{2}\right)
$$

Substitution of (8) into the Schrödinder equation (4) yields a closed equation for $\psi$,

$$
\frac{1}{2 m^{*}}\left(i \hbar \nabla+e^{*} \mathbf{A}\right)^{2} \psi+4 D|\psi|^{2} \psi-4 D\left|\psi_{\infty}\right|^{2} \psi=0 .
$$

One can see that for $4 D=\beta$ and the standard GL relation, $\alpha=-\beta\left|\psi_{\infty}\right|^{2}$, Eq. (9) turns into the GL equation,

$$
\frac{1}{2 m^{*}}\left(i \hbar \nabla+e^{*} \mathbf{A}\right)^{2} \psi+\beta|\psi|^{2} \psi+\alpha \psi=0 \text {. }
$$

With $D=\beta / 4$, Eq. (8) coincides with Eq. (3).

Now we show that the charge neutrality is a good approximation in the general case. A simple estimate shows that the deviation from charge neutrality, $\rho=-\varepsilon \nabla^{2} \varphi$, is much smaller than the increase of the normal electron charge density $-4 e^{2} \beta^{-1} \varphi$. Indeed, the potential varies on the scale of the coherence length, $\nabla^{2} \varphi \sim \xi^{-2} \varphi$, therefore the ratio of these two charge densities behaves as

$$
\frac{\varepsilon \nabla^{2} \varphi}{4 e^{2} \beta^{-1} \varphi} \sim \frac{\beta \rho_{\mathrm{S}}^{\infty} \kappa^{2}}{e m c^{2}} \sim \frac{k_{\mathrm{B}}^{2} T_{c}^{2} \kappa^{2}}{\epsilon_{F} m c^{2}} \frac{\left|\rho_{\mathrm{S}}^{\infty}\right|}{\left|\rho_{\text {lattice }}\right|} \sim \frac{\hbar^{2} \lambda^{2}}{m^{2} c^{2} \xi^{4}}
$$

Here we have used the BCS value of $\beta$ [9], see Eq. (13]), and introduced the GL parameter $\kappa=\lambda / \xi$. Even for extreme values of the high- $T_{c}$ materials $\left[\kappa \sim 100, T_{c} \sim\right.$ $90 \mathrm{~K}$, the Fermi energy $\epsilon_{F} \sim 0.08 \mathrm{eV}, m \sim 4 m_{e}, \varepsilon=4 \varepsilon_{0}$, $\left.\rho_{\mathrm{s}}^{\infty} \sim-\rho_{\text {lattice }}\right]$ the estimate (11) gives about $10^{-5}$. For conventional superconductors the ratio (11) is typically about $10^{-9}$. Accordingly, the deviation from the charge neutrality may be disregarded when one solves for the wave function. With $\varepsilon \nabla^{2} \varphi \approx 0$ the Poisson equation (7) simplifies to Eq. (3).

Now we compare the above result with alternative approaches. First, far from the core one can make the local approximation of the Ginzburg-Landau equation (10)

$$
\frac{m^{*} v^{2}}{2} \psi+\beta|\psi|^{2} \psi+\alpha \psi=0
$$

where the velocity is given by the current, $\mathbf{j}=e^{*} \mathbf{v}|\psi|^{2}$. One can see that in this limit the electrostatic potential found from (12) and (8) equals the Bernoulli potential (2). We note that the incompressible superconducting liquid discussed by London [1] corresponds to the limit $\kappa \rightarrow \infty$ of the GL theory 8 . In this limit, the local approximation is exact.

Second, formula (3) is similar to the formula proposed by Khomskii and Kusmartsev [10,11], $\varphi_{\mathrm{KK}}=\Delta^{2} /\left(4 e \epsilon_{F}\right)$. It turns out, however, that from the BCS-Gor'kov relations [9],

$$
\beta=\frac{24\left(\pi k_{\mathrm{B}} T_{c}\right)^{2}}{7 \zeta(3) \epsilon_{F} N}, \quad \psi=\frac{\sqrt{7 \zeta(3) N}}{4 \pi k_{\mathrm{B}} T_{c}} \Delta,
$$

one obtains

$$
\frac{\beta}{2 e}|\psi|^{2}=\frac{3 \Delta^{2}}{4 e \epsilon_{F}}
$$

i.e., our value is three times larger than $\varphi_{\mathrm{KK}}$. The similarity of (14) with the potential of Khomskii and Kusmartsev is, however, accidental. While (14) follows from the intertial and Lorentz forces, the potential $\varphi_{\mathrm{KK}}$ results from the condensation energy of the pairing. We have neglected the condensation energy in our treatment.

In the rest of this paper we show actual charge profiles of an isolated vortex and of the vortex lattice. We use known solutions of the Ginzburg-Landau equation and evaluate the electrostatic potential $\varphi$ from Eq. (3). The deviation of the charge density from neutrality then follows from the Poisson equation, $\rho=-\varepsilon \nabla^{2} \varphi$.

We start the discussion using the isolated vortex model suggested by Clem [12],

$$
|\psi|^{2}=\left|\psi_{\infty}\right|^{2} \frac{r^{2}}{r^{2}+\xi_{\mathrm{v}}^{2}},
$$

where $\xi_{\mathrm{v}} \sim \xi$ is given by the minimum of the free energy as $\xi_{\mathrm{v}}=\xi \sqrt{2} \sqrt{1-K_{0}^{2}\left(\xi_{\mathrm{v}} / \lambda\right) / K_{1}^{2}\left(\xi_{\mathrm{v}} / \lambda\right)}$, where $K_{0}$ and $K_{1}$ are modified Bessell functions. This wave function fails at long distances, $r \sim \lambda$, where the correct behavior follows the local approximation, $|\psi|^{2}=\left|\psi_{\infty}\right|^{2}\left(1+\frac{m^{*} v^{2}}{2 \alpha}\right)$, but it provides a good approximation in the vicinity of the core, $r \sim \xi$, where the dominant charge contribution is located. The electric field, $\mathbf{E}=-\nabla \varphi$, obtained from (15) and (3) is directed radially,

$$
\mathbf{E}=-\frac{\beta}{e}\left|\psi_{\infty}\right|^{2} \frac{\xi_{\mathrm{v}}^{2}}{\left(r^{2}+\xi_{\mathrm{v}}^{2}\right)^{2}} \mathbf{r}
$$


The charge density corresponding to (15),

$$
\begin{aligned}
\rho & =-2 \varepsilon \frac{\beta}{e}\left|\psi_{\infty}\right|^{2} \frac{\xi_{\mathrm{v}}^{2}\left(\xi_{\mathrm{v}}^{2}-r^{2}\right)}{\left(r^{2}+\xi_{\mathrm{v}}^{2}\right)^{3}} \\
& =-\frac{a_{\mathrm{B}} e}{\pi} \frac{\xi_{\mathrm{v}}^{2}}{2 \xi^{2}} \frac{\xi_{\mathrm{v}}^{2}-r^{2}}{\left(r^{2}+\xi_{\mathrm{v}}^{2}\right)^{3}},
\end{aligned}
$$

is depleted in the core, $r<\xi_{\mathrm{v}}$, and enhanced outside the core, $r>\xi_{\mathrm{v}}$. Here, $a_{\mathrm{B}}=\frac{4 \pi \varepsilon \hbar^{2}}{m e^{2}}$ is the Bohr radius in the crystal.

The second form of 17 can be compared with the result of Blatter et al. 4 , who found a more complicated factor, $\rho_{\mathrm{Bl}} \approx \rho \times \pi^{-2} \frac{d T_{c}}{d \ln \epsilon_{F}}$. We note that Blatter et al. approached the problem from the microscopic side using the BCS relation between the total charge and the chemical potential. This charge is moreover screened in the spirit of Jakeman and Pike 13] within the Thomas-Fermi approximation. Since $\frac{d T_{c}}{d \ln \epsilon_{F}} \approx \ln \left(\hbar \omega_{D} / T_{c}\right) \sim 1$ to 10 , their numerical results are close to ours.

The charge of the vortex core per unit length, $Q=$ $2 \pi \int_{0}^{\xi_{\mathrm{v}}} r \rho \mathrm{d} r=2 \pi \varepsilon E\left(\xi_{\mathrm{v}}\right) \xi_{\mathrm{v}}$, results as

$$
Q=-\pi \varepsilon \frac{\beta}{2 e}\left|\psi_{\infty}\right|^{2}=\frac{\pi \varepsilon \alpha}{2 e}=-\frac{a_{\mathrm{B}} e}{4 \xi^{2}} .
$$

In a single pancake (a vortex of length $d=1.17 \cdot 10^{-9} \mathrm{~m}$ ) in $\mathrm{YBa}_{2} \mathrm{Cu}_{3} \mathrm{O}_{7-\delta}(\mathrm{YBCO})$ at $77 \mathrm{~K}$, the depleted charge is $Q * d=-0.004 \mathrm{e}$. We have used $a_{\mathrm{B}}=0.05 \mathrm{~nm}$ and $\xi=$ $1.91 \mathrm{~nm}$, where the coherence lenght follows from $\xi=\lambda / \kappa$ with $\lambda=191 \mathrm{~nm}$ and $\kappa=100$. With the quasiparticle screening in the spirit of van Vijfeijken and Staas [14], this value will be reduced by $\rho_{\mathrm{s}} / \rho=2\left|\psi_{\infty}\right|^{2} / N \approx 1-$ $\left(T / T_{c}\right)^{4}=0.5$ for $T=77 \mathrm{~K}$.

Note that the vortex charge 18 has a value independent of the core diameter $\xi_{\mathrm{v}}$. A similar value, $Q=$ $-1.28 \times a_{\mathrm{B}} e /\left(4 \xi^{2}\right)$, results for a different model function, $|\psi|=\left|\psi_{\infty}\right| \tanh (r / \xi)$, recommended in [8]. This seems to contradict the expectation from the local approximation, where the core diameter plays a dominant role. In the local approach, the velocity close to the core reads $v \approx \frac{e \Phi_{0}}{2 \pi m r}$. From the Bernoulli potential (2) and the Poisson equation one finds that inside the cylinder of radius $r$ the local approximation predicts a charge

$$
Q_{\mathrm{la}}=-\frac{e \varepsilon \Phi_{0}^{2}}{2 \pi m r^{2}}
$$

With the core radius equal to the coherence length, $r=\xi=\hbar / \sqrt{-2 m^{*} \alpha}$, the classical estimate (19) reproduces the nonlocal result (18). Although the exact agreement of Eq. (19) with Eq. (18) is rather accidental, it shows that classical estimates used in the literature are reasonable.

An example of the electrostatic potential for the triangular vortex lattice is presented in Fig. 1. One can see that in the core region the potential has a shape similar to the Clem model. The maximum amplitude, $\varphi_{\max }=-1.3 \mathrm{mV}$, is identical to the maximum potential obtained from the Clem model since the GL function $|\psi|^{2}$ reaches zero at the vortex center in both cases. The GL function used in Fig. 1 has been evaluated by the iteration method described in 15$]$.

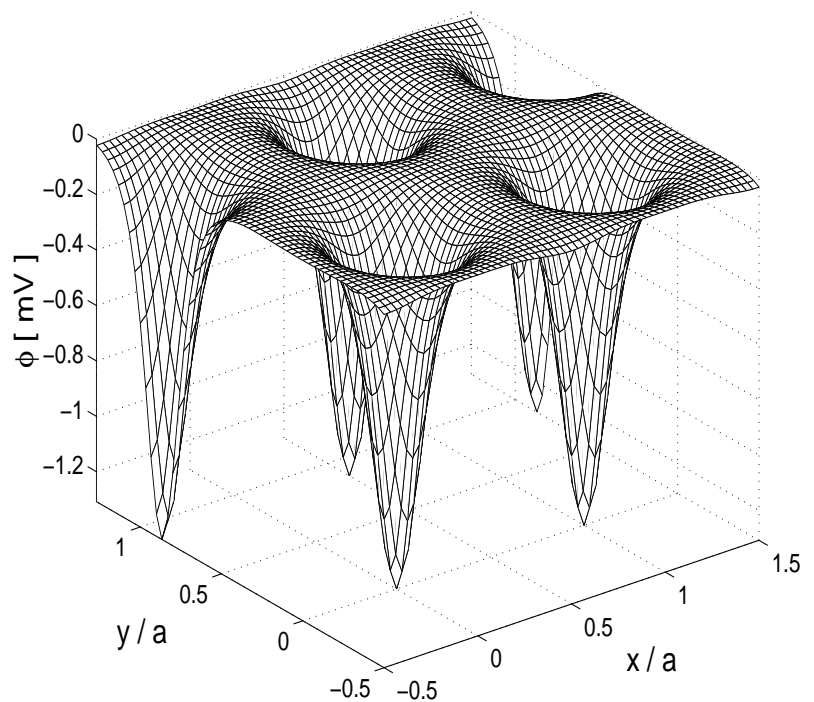

FIG. 1. Electrostatic potential $\varphi$ in a superconductor with triangular vortex lattice of spacing $a$. The shape of the potential follows the magnitude of the GL function, see Eq. (3), which depends exclusively on the reduced induction $b=B / B_{c 2}=0.05$ and the GL parameter $\kappa=100$. The presented magnitude of $\varphi$ results for $\lambda=191 \mathrm{~nm}$ and $m=4 m_{0}$, which corresponds to $\mathrm{YBCO}$ at temperature $77 \mathrm{~K}$.

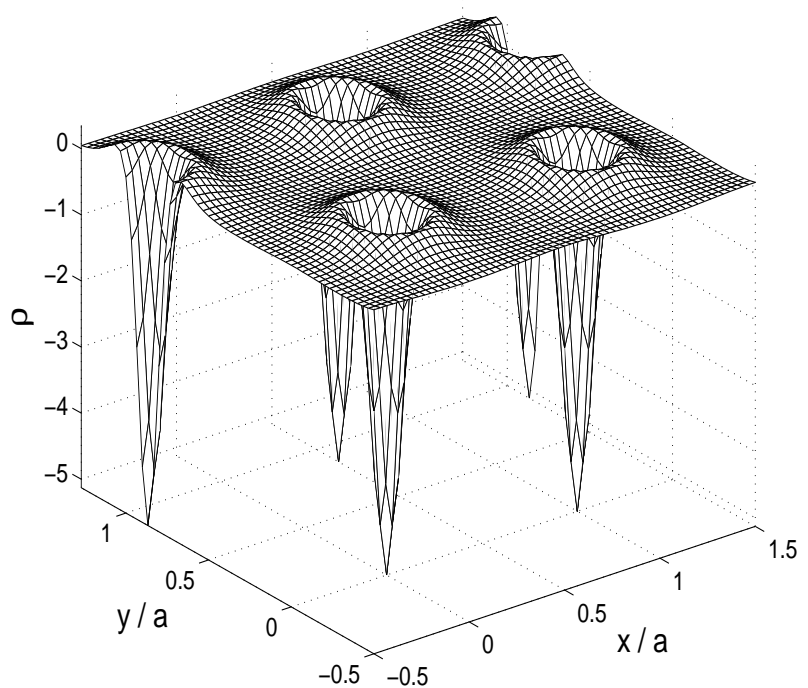

FIG. 2. Charge density $\rho=-\varepsilon \nabla^{2} \varphi$ in a superconductor with a triangular vortex lattice. All the parameters are the same as in Fig. 11. The charge density is plotted in units $10^{-6}$ electrons per unit cell, with the volume of the unit cell $0.173 \mathrm{~nm}^{3}$ corresponding to YBCO.

The density of charge corresponding to the potential in Fig. 1 is shown in Fig. 2. In the core, the charge is nega- 
tive, i.e., the density of charge carriers is depleted. Outside the core, the charge is positive, i.e. there is charge built up which screens the charge of the core. The radius of the vortex core (radius at which the charge changes sign) found in Fig. 2 is $1.53 \xi$, comparable to the value $1.41 \xi$ obtained from the Clem model. The maximum of the screening charge appears at $2.4 \xi$, while the Clem model yields $2 \xi$. The maximum of the depleted charge in the core centre is $-5 \cdot 10^{-6}$ electron/cell while the Clem model gives $-6.8 \cdot 10^{-6}$ electron/cell. The core charge per unit lenght is thus reduced to $85 \%$ of the Clem model. These numerical results thus show that the approximation based on the Clem model is reasonable inside and in the vicinity of the core.

In conclusion, we have shown that the GL function can be used to evaluate the electric field in superconductors, see Eq. (3), in spite of the fact that the electrostatic potential is absent in the GL theory. For convenient estimates of the core charge we have derived the simple formula (18). Our theory neglects contributions from the condensation energy and the screening by normal particles; more work on this is under way. Due to the neglected screening, the theory applies only for intermediate temperatures where the GL theory still gives reasonable results while the share of the superconducting electrons is already of the order of unity. Numerical results relevant to $\mathrm{YBCO}$ at the temperature $77 \mathrm{~K}$ and at low magnetic field with well separated vortex cores have been presented.

This work was supported by MŠMT program Kontakt ME160 and GAČR 202000643, GAAV A1010806 and A1010919 grants. The European ESF program VORTEX is gratefully acknowledged.

[1] F. London, Superfluids, vol. I, Sec. 8 (Willey, New York, 1950).

[2] M. A. R. LeBlanc, Supercond. Sci. Technol. 10, 929 (1997).

[3] Y. Matsuda and K. Kumagai, to be published.

[4] G. Blatter, M. Fiegel'man, V. Geshkenbein, A. Larkin and A. van Otterlo, Phys. Rev. Lett. 77, 566 (1996).

[5] T. M. Mishonov, cond-mat/0004286.

[6] M. V. Feigel'man, V. B. Geshkenbein, A. I. Larkin and V. M. Vinokur, Pisma Zh. Eksp. Teor. Fiz. 62, 811 (1995) [JETP Lett. 62, 835 (1995)].

[7] E. H. Brandt, Phys. Lett. A 39, 227 (1972).

[8] M. Tinkham, Introduction to superconductivity, Secs. 4 and 5 (McGraw-Hill, New York,1996).

[9] L. P. Gor'kov, Zh. Eksperim. i Teor. Fiz. 36, 1918 (1959) [Sov. Phys.-JETP 9, 1364 (1959)].

[10] D. I. Khomskii and F. V. Kusmartsev, Phys. Rev. B 46, 14245 (1992).
[11] D. I. Khomskii and A. Freimuth, Phys. Rev. Lett. 75, 1384 (1995).

[12] J. R. Clem, Journal of Low Temp. Phys. 18, 427 (1975).

[13] E. Jakeman and E. R. Pike, Proc. Phys. Soc. 91, 422 (1967).

[14] A. G. van Vijfeijken and F. S. Staas, Phys. Lett. 12, 175 (1964).

[15] E. H. Brandt, Phys. Rev. Lett. 78, 2208 (1997). 